\title{
Coupled Simulation of a Room Air-conditioner with CFD Models for Indoor Environment
}

\author{
Hongtao Qiao $^{1^{*}} \quad \mathrm{Xu} \mathrm{Han}^{2} \quad$ Saleh Nabi $^{1} \quad$ Christopher R. Laughman $^{1}$ \\ ${ }^{1}$ Mitsubishi Electric Research Laboratories, USA, \{qiao, nabi, laughman $\}$ @merl . com \\ ${ }^{2}$ University of Colorado Boulder, USA, Xu.Han-2 @ colorado . edu
}

\begin{abstract}
Coupled simulation of building energy systems (BES) and computation fluid dynamics (CFD) often focuses on the integration of air handlers with indoor environment, and does not incorporate vapor compression systems into the analysis, yielding inaccurate prediction of building energy consumption. This paper presents a coupled simulation to explore the pull-down performance of a room air conditioning system. The dynamic models of the air-conditioner are constructed in Modelica, whereas the indoor environment is simulated in OpenFOAM. Dynamic characteristics will be compared with different vane angles and airflow modes. Numerical simulations demonstrate that both vane angle and airflow mode exhibit pronounced impact on the pull-down time. Meanwhile, the well-mixed assumption that most of building energy simulation programs are built upon exhibits drastically different dynamic characteristics compared to the detailed CFD model, suggesting that neglecting non-uniform air flow and temperature distributions in buildings might lead to significant errors in control design.
\end{abstract}

Keywords: Modelica, OpenFOAM, co-simulation, building energy simulation, vapor compression system

\section{Introduction}

Buildings account for $40 \%$ of the primary energy in the U.S. and are important sources of $\mathrm{CO}_{2}$ emission. Currently, roughly half of energy consumption in buildings is used by spacing heating and cooling. These end-uses represent significant opportunities to reduce energy consumption, improve energy security and reduce $\mathrm{CO}_{2}$ emissions. Therefore, efficiency improvements in HVAC systems become more enticing and will play a crucial role in energy revolution.

Due to the multi-scale, highly nonlinear and complex nature of their dynamic characteristics, design of high energy-efficient HVAC systems nowadays heavily relies on computer simulations because they can not only provide verifiable and robust predictions of system performance, but also radically accelerate the design, testing, and specification of these systems by drastically reducing the number of experimental iterations required. Meanwhile, model-based design approach is often used to develop and evaluate advanced control algorithms of HVAC systems.

The assessment of closed-loop control performance of HVAC systems often requires an integrated approach that couples the dynamic models of HAVC system with building energy simulation programs. Most of these building simulation programs assume that indoor air is well mixed in order to simplify the computation. However, this prevailing assumption fails to simulate the distribution of temperature, pressure, concentration in buildings with large space and high heat gain. As a result, these programs cannot accurately predict building energy consumption and the closed-loop performance of HVAC systems. In addition, they cannot satisfy advanced design requirements, such as personal cooling/heating in occupied zone and optimal sensor placement, due to lack of local thermal comfort information (Zhai et al., 2002).

In contrast with the well-mixed assumption, computational fluid dynamics (CFD) divides fluid domain into a large number of small volumes such that a detailed prediction of airflow and temperature distributions, thermal comfort and indoor air quality can be obtained. Consequently, coupling building energy simulation programs with CFD can be effective to overcome the deficiencies of stand-alone programs and achieve better results. In such a way, building simulations can provide dynamic boundary conditions to CFD, whereas CFD simulates the airflow dynamics based on these boundary conditions and then can send local airflow and temperature information back to building simulations such that the closed-loop performance of HVAC systems can be evaluated.

Recently, there is an emerging interest in using fast fluid dynamics (FFD) for indoor environment simulation instead of full-scale CFD because of superior computational speed of FFD (Zuo et al., 2016). Compared with CFD, however, FFD possesses many limitations. First of all, FFD does not support unstructured mesh and thus cannot deal with complex geometry. Meanwhile, FFD does not have turbulence flow models, which may yield inaccurate predictions in airflow dynamics. Moreover, FFD assumes that heat flow is uniformly injected into space and therefore cannot handle non-uniform heat source/sink. All these 
limitations impose great challenges when applying FFD in indoor environment simulations.

Comprehensive review of the literature regarding the coupled simulation of building energy systems and the indoor environment (Tian et al., 2018) indicates that the previous studies only focused on the integration of air handler units with indoor environment, whereas none of these studies ever took into account the dynamics of vapor compression systems, which are the essential part of building HVAC systems through which heat is removed from or added to buildings. Also, they are the primary energy consumer compared to other auxiliary components, such as pumps and fans. Without incorporating vapor compression systems into the coupled analysis, it is impossible to accurately predict building energy consumption. Meanwhile, feedback control design of such systems requires to account for the complex dynamic characteristics of phase-changing refrigerant flow, which are affected by air flows inside buildings. Therefore, it is worthwhile to couple vapor compression system with indoor environment to explore the closed-loop dynamic performance of these systems.

Another interesting finding through literature review is that the reported coupled simulations are all based on the commercial CFD programs, e.g., Fluent, CFX and STAR-CD, rather than open-source code. These CFD programs are extremely expensive and might not be readily available for everyone. Development and maintenance of the middleware that couples the commercial CFD programs with building energy simulations could be costly and time-consuming since the source code is not generally accessible. As an opensource and well recognized CFD engine, OpenFOAM possesses many advantages compared to the commercial programs because of unrestricted access to its source code. Full control on OpenFOAM simulator makes integration with other programs through middleware much easier.

To bridge the identified research gap, this paper attempts to couple the dynamic models of a wallmounted split-type air-conditioner with the detailed CFD model for indoor environment to explore its closed-loop pull-down performance with different vane angles and airflow modes. The dynamic models of the air-conditioner are constructed in Modelica, whereas the indoor environment is simulated in OpenFOAM. Therefore, a co-simulation platform that couples Modelica with OpenFOAM needs to be developed. Dynamic characteristics will be compared against those obtained by the well-mixed air model to demonstrate the effect of non-uniform airflow distribution on the system performance.

The remainder of the paper is organized as follows. In Section 2, the coupling mechanisms between Modelica and OpenFOAM are presented. In Section 3, we describe the dynamic models for the air-conditioning system and the models for air flow dynamics. In Section
4, we discuss the influence of the vane angle and airflow mode on the pull-down performance of the airconditioning system. Conclusions from this work are then summarized in Section 5.

\section{Coupling Mechanisms}

A middleware interface is required to facilitate data exchange between individual programs, i.e., Modelica and OpenFOAM in this study. A quasi-dynamic data synchronization scheme is used in the coupled simulation, which means that the received data of individual programs are discrete with time and remain unchanged between two successive synchronization points, and will be only updated when the next synchronization point is reached. The synchronization time step needs to be predefined before simulation, and cannot change during simulation. Please be noted that both Modelica and OpenFOAM have their respective integration time steps, which can be either fixed or adaptive and are usually much smaller than the synchronization time step.

Zuo et al. (2016) developed a coupled simulation between FFD and the Modelica Buildings library for the dynamic ventilation system with stratified air distribution. In order to make life easier, we decided to modify their framework so that OpenFOAM can communicate with Modelica. Therefore, the implementation on the Modelica side and between Modelica and middleware requires no modifications. Different from that Modelica uses external $\mathrm{C}$ functions to exchange data with the middleware, OpenFOAM uses its built-in externalCoupled function object which provides a file-based communication interface to transfer data to and from OpenFOAM (OpenFOAM Foundation, 2017), as shown in Fig. 1. The data exchange employs specialized boundary conditions to provide either uni- or bi-directional models. At start-up, the externalcoupled function creates a lock file, i.e., OpenFOAM. lock, to signal the external source, i.e., middleware in this case, to wait. During the boundary condition update, boundary values are written to file, e.g., data. out. The lock file is then removed, instructing the external source to take control of the program execution. When ready, the external program should create the return values to file, e.g., data.in, and then re-instate the lock file. The externalcoupled function will then read the return values, and pass program execution back to OpenFOAM. The logic of data exchange between Modelica and OpenFOAM is shown in Fig. 2. 


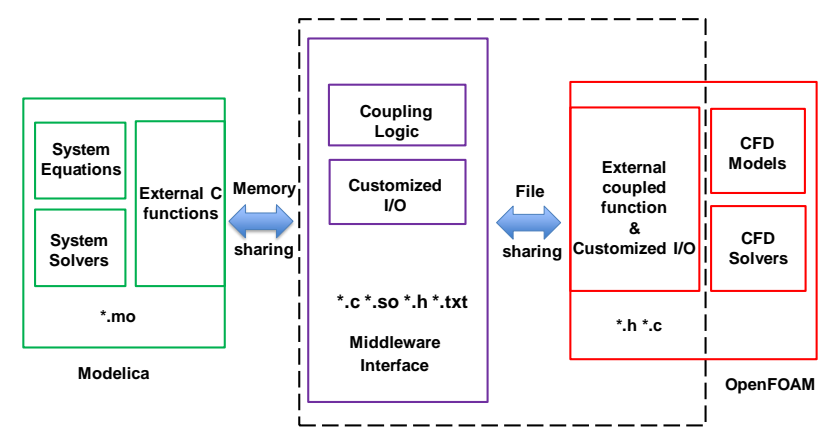

Figure 1. Coupling between Modelica and OpenFOAM.

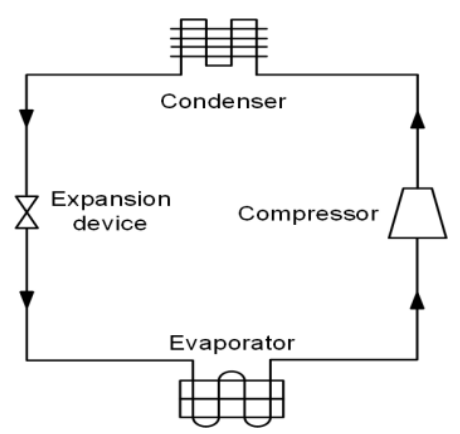

Figure 3. Schematic of an air-conditioning system.

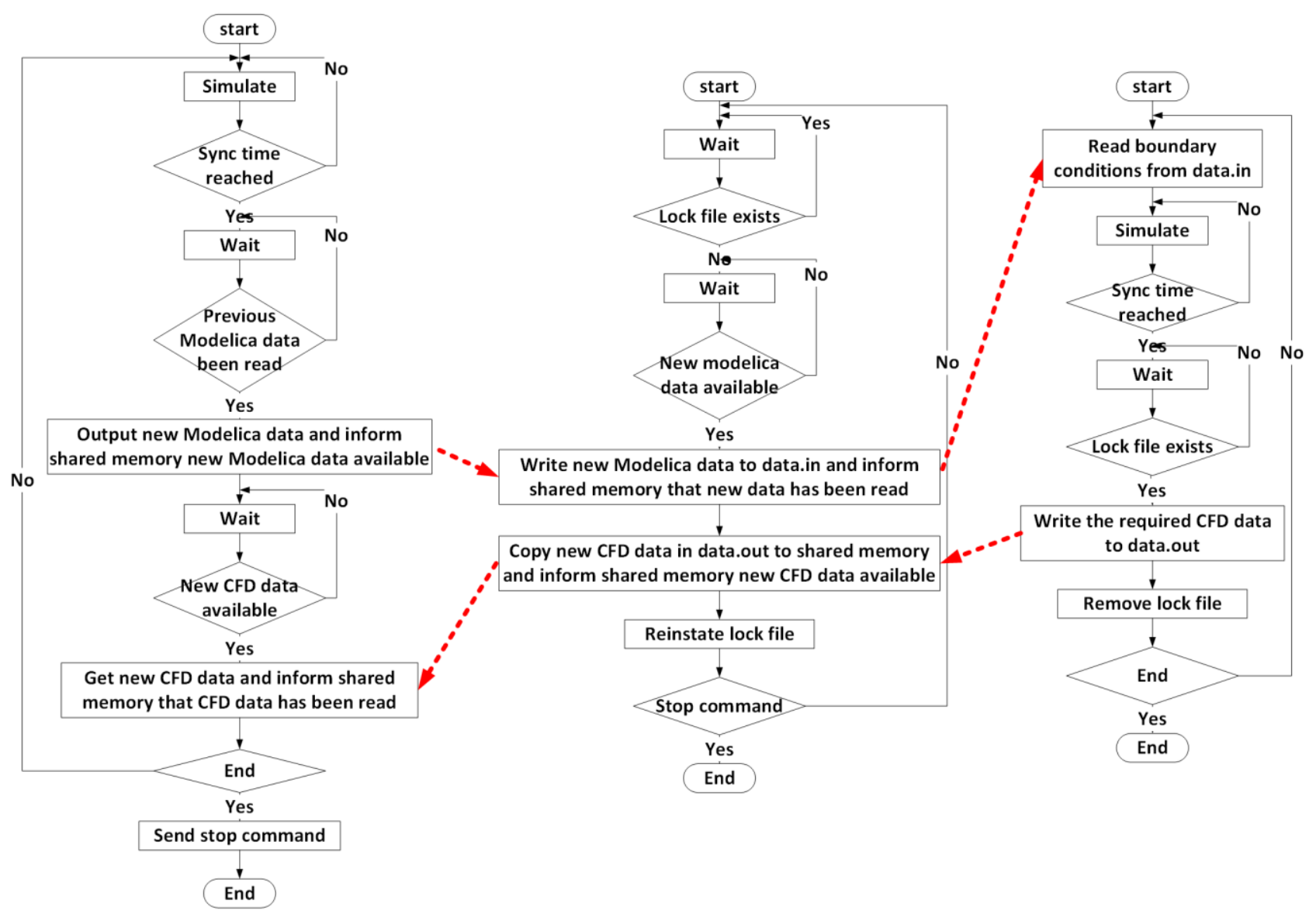

Figure 2. Logic of data exchange in co-simulation.

\section{Model Development}

The studied air-conditioning system consists of two main parts: the outdoor unit and the indoor unit. The outdoor unit is installed on or near the wall outside of the room or space that you wish to cool. The outdoor unit houses the compressor, condenser, whereas the indoor unit contains the evaporator, expansion device, a blower fan and an air filter. Fig. 3 illustrates the schematic of a typical air-conditioning system.

\subsection{Compressor Model}

A variable-speed low-side scroll compressor, in which the motor is cooled by compressed low-pressure suction refrigerant, was used in this work. Because the performance map for the compressor has reduced accuracy when extrapolated beyond the specific ranges of saturated discharge and suction temperatures over which it is tabulated, we converted the performance map into a set of curve-fitted equations to avoid poor numerical behavior during the simulations.

The volumetric efficiency of this compressor model is a function of the suction pressure, discharge pressure and compressor frequency

$$
\eta_{\mathrm{v}}=\kappa_{0}+\kappa_{1} \varphi+\kappa_{2} \varphi^{2}+\kappa_{3}\left(p_{\text {dis }}-p_{\text {suc }}\right)\left(1+\kappa_{4} p_{\text {suc }}\right)
$$


where $\varphi=p_{\text {did }} / p_{\text {suc }}, \kappa_{\mathrm{i}}=\mathrm{a}_{\mathrm{i}, 1}+\mathrm{a}_{\mathrm{i}, 2} \varpi+\mathrm{a}_{\mathrm{i}, 3} \varpi^{2}$, and $\varpi=f l f_{\text {nom. }}$. The power consumed by the compressor is determined by

$$
\dot{W}=\zeta_{1} p_{\text {suc }} \dot{V}_{\text {suc }}\left(\varphi^{\zeta_{2}}-\zeta_{3}\right)+\zeta_{4}
$$

where $\zeta_{\mathrm{i}}=\mathrm{b}_{\mathrm{i}, 1}+\mathrm{b}_{\mathrm{i}, 2} \varpi+\mathrm{b}_{\mathrm{i}, 3} \varpi^{2}$. In addition, the fraction of the compressor power absorbed by the refrigeration is determined by

$$
\lambda=\theta_{0}+\theta_{1} \varphi+\theta_{2} \varphi^{2}
$$

where $\theta_{\mathrm{i}}=\mathrm{c}_{\mathrm{i}, 1}+\mathrm{c}_{\mathrm{i}, 2} \varpi+\mathrm{c}_{\mathrm{i}, 3} \varpi^{2}$. Lastly, the mass flow rate delivered by the compressor is determined by

$$
\dot{m}=\eta_{v} f \rho_{s u c} V_{\text {disp }}
$$

and the enthalpy of the discharged refrigerant is

$$
h_{d i s}=\lambda \dot{W} / \dot{m}+h_{s u c}
$$

\subsection{Expansion Valve Model}

Linear electronic expansion valves (LEVs) were also used in the system. A standard orifice-type relationship between mass flow rate and pressure drop across the valve was used to describe the system behaviour, in which the mass flow rate through the valve is determined by the flow coefficient, the inlet density, and the pressure difference across the valve

$$
\dot{m}=C_{v} \sqrt{\rho_{\text {in }} \Delta p}
$$

where the flow coefficient $C_{v}$ is a function of the valve opening determined by regression based on experimental data.

\subsection{Heat Exchanger Models}

The heat exchangers were described by a finite-volume model of the heat and fluid flow. Each heat exchanger segment is divided into three sections: the refrigerant stream, the finned walls, and the air stream. The refrigerant stream is described by a one-dimensional flow with fluid properties varying only in the direction of flow; consequently, these properties are uniform or averaged at every cross section along the axis of the channel. Additional assumptions used to simplify the dynamic models included: (1) the fluid is Newtonian, (2) axial heat conduction in the direction of refrigerant flow is ignored, (3) viscous dissipation is neglected, (4) liquid and vapor are in thermodynamic equilibrium in each volume in the two-phase region, (5) the potential energy and kinetic energy of the refrigerant are neglected, and (6) dynamic pressure waves are of minor importance and are thus neglected in the momentum equation (Brasz and Koenig, 1983). The conservation laws can thus be formulated as follows:

$$
\begin{aligned}
& \frac{\partial}{\partial t}(\bar{\rho} A)+\frac{\partial}{\partial z}(G A)=0 \\
& \frac{\partial}{\partial t}\left(\bar{\rho} \bar{h}_{\rho} A\right)+\frac{\partial}{\partial z}(G \bar{h} A)=P q_{w}^{\prime \prime}+\frac{\partial}{\partial t}(p A) \\
& A \frac{\partial p}{\partial z}+\bar{\tau}_{w} P=0
\end{aligned}
$$

where average density, average mass flux, average density-weighted enthalpy, average flow-weighted enthalpy, and average wall shear stress are defined as follows, respectively.

$$
\begin{gathered}
\bar{\rho}=\frac{1}{A} \int_{A} \rho d A, G=\frac{1}{A} \int_{A} \rho u d A, \bar{h}_{\rho}=\frac{1}{\bar{\rho}}\left(\frac{1}{A} \int_{A} \rho h d A\right), \\
\bar{h}=\frac{1}{G}\left(\frac{1}{A} \int_{A} \rho u h d A\right), \bar{\tau}_{w}=\frac{1}{P} \int_{P} \tau_{w} d l .
\end{gathered}
$$

For single-phase flow, the refrigerant density can be presumed to be uniform at each cross sectional area, and the following relations can be readily obtained

$\bar{\rho}=\rho$

$\bar{h}_{\rho}=\bar{h}=h$

This work relaxed the typical homogeneous flow assumption of equality between the velocities of vapor and liquid for control volumes in the two-phase region to achieve a more realistic refrigerant system mass inventory for the overall system. This necessitated the use of the average density and specific enthalpies for two-phase flow, which are determined by

$$
\begin{aligned}
& \bar{\rho}=\rho_{g} \gamma+\rho_{f}(1-\gamma) \\
& \bar{h}_{\rho}=\left[\rho_{g} h_{g} \gamma+\rho_{f} h_{f}(1-\gamma)\right] / \bar{\rho} \\
& \bar{h}=h_{g} x+h_{f}(1-x)
\end{aligned}
$$

where the void fraction and flow quality are defined as

$\gamma=\frac{A_{g}}{A}$

$x=\frac{\rho_{g} u_{g} \gamma}{G}$

The flow quality $x$ is distinct from the static quality $\hat{x}$, which is defined as the ratio of mass of vapor to that of the total mixture,

$$
\hat{x}=\frac{M_{g}}{M_{g}+M_{f}}=\frac{\rho_{g} \gamma}{\rho_{g} \gamma+\rho_{f}(1-\gamma)}
$$

Hence, the density-weighted enthalpy can be described using the static quality

$$
\bar{h}_{\rho}=\hat{x} h_{g}+(1-\hat{x}) h_{f}
$$

The pressures and density-weighted enthalpies of each control volume are used as the state variables in the heat exchanger model. Density-weighted enthalpy can be related to flow-weighted enthalpy via

$$
\bar{h}-\bar{h}_{\rho}=(x-\hat{x})\left(h_{g}-h_{f}\right)
$$

Note that $x$ and $\hat{x}$ are both zero for the subcooled liquid and unity for the superheated vapor, implying that these two enthalpies should be equal to each other for single-phase flows. All of the variables in Eq. (19) are thermodynamic properties, and can be readily calculated except for the flow quality $x$.

While this refrigerant-side model was principally developed for the heat exchangers, it was also used to describe the behavior of the refrigerant pipes connecting the components. These pipe models were an important part of the overall system model because they contained a significant fraction of the refrigerant in the entire 
system. The main distinction between the pipe models and the heat exchanger models regards the air-side models used: the heat exchanger air-side models describe the effect of the enhanced heat transfer surface, while the pipe air-side models use natural convection as the boundary condition.

The model of the air-side of the heat exchanger is based on the following assumptions: (1) onedimensional quasi-steady airflows, (2) negligible heat conduction in direction of the air flow, (3) the temperature profile within fins follows the steady-state profile, allowing the use of heat transfer and combined heat and mass transfer fin efficiencies, (4) simultaneous heat and mass transfer follows the Lewis analogy, and (5) equal temperature of tube wall and fins within each control volume. Under these assumptions, the governing equations for the air side describing the energy balances for the wetted coils are

$$
\begin{aligned}
& \dot{m}_{a} c_{p, a} \frac{d T_{a}}{d y} \Delta y=\alpha_{a}\left(A_{o, t}+\eta_{f i n} A_{o, f i n}\right)\left(T_{w}-T_{a}\right) \\
& \dot{m}_{a} \frac{d \omega_{a}}{d y} \Delta y=\alpha_{m}\left(A_{o, t}+\eta_{f i n} A_{o, f i n}\right) \min \left(0, \omega_{\mathrm{w}, s a t}-\omega_{a}\right)
\end{aligned}
$$

where $\omega_{\mathrm{w} \text {,sat }}$ is the humidity ratio of saturated air evaluated at $T_{\mathrm{w}}$. The mass transfer coefficient $\alpha_{\mathrm{m}}$ is determined by applying the Lewis analogy.

Finally, without considering the axial conduction along the tube, the energy equation of the tube walls and associated fins can be written as

$$
\begin{aligned}
& \left(M_{t} c_{p, t}+M_{\text {fin }} c_{p, \text { fin }}\right) \frac{d T_{w}}{d t}=q_{r}+q_{a} \\
& q_{r}=\alpha_{r} A\left(T_{r}-T_{w}\right) \\
& q_{a}=\dot{m}_{a}\left[c_{p, a}\left(T_{a, \text { in }}-T_{a, \text { out }}\right)+\left(\omega_{a, \text { in }}-\omega_{a, \text { out }}\right) \Delta h_{f g}\right]
\end{aligned}
$$

\subsection{CFD Model}

CFD uses numerical simulations to predict the fluid flow phenomena based on the conservation laws (conservation of mass, momentum and energy) governing fluid motion. The general conservation equation of any quantity $\phi$ is given by

$$
\frac{\partial}{\partial t}(\rho \phi)=-\nabla \bullet(\rho \mathrm{U} \phi)+\nabla \bullet(D \nabla \phi)+S_{\phi}
$$

In the equation above, $D$ stands for the diffusion coefficient, that can be a scalar or a vector and $S_{\phi}$ stands for any kind of sources or sinks that influence the quantity $\phi$. Now we are able to simply derive the mass, momentum and other conservative equations out of this by replacing the quantity $\phi$ by the quantity of interest.

The partial differential conservation equations are more complex than they appear and they are non-linear, coupled, and difficult to solve. Therefore, discretization methods, e.g., finite volume method, are used to approximate the differential equations by a system of algebraic equations, which can be solved on a computer. The approximations are applied to small domains in space and/or time so the numerical solution provides results in discrete locations in space and time.

\section{Case Study}

The objective of this numerical study is to explore the dynamic performance of a wall-mounted split-type airconditioner with different vane angles and airflow modes during pull-down operation. The air conditioning system used R410A as the working fluid. The compressor was a low-side scroll compressor with displacement of $6.8 \mathrm{~cm}^{3}$ and nominal rotational speed of $3500 \mathrm{rpm}$, and both heat exchangers were louvered finand-tube heat exchangers. The heat exchanger models were augmented by a set of empirical closure relations describing the single- and two-phase heat transfer coefficients and frictional pressure drops for the fluid on both the refrigerant side and the air-side (Qiao et al., 2015). The Levy void fraction model (Levy, 1967) was used to compute the two-phase refrigerant mass inventory. A tube-by-tube approach was employed for the heat exchanger analysis, i.e., the performance of each tube was analyzed separately and each tube was associated with different refrigerant and air parameters.

Two PI controllers in the air-conditioning system adjust the EEV opening and compressor speed to control the suction superheat at $3 \mathrm{~K}$ and the return air temperature at $26^{\circ} \mathrm{C}$, respectively. The values of PI gains are given in Table 1.

The indoor unit is installed at the center of the wall in a room with dimensions of $5 \times 5 \times 2.6 \mathrm{~m}$ (length $\times$ width $\times$ height), as shown in Fig. 4. The solver settings and boundary conditions for the CFD room model are summarized in Table 2. To reduce the calculation load, the indoor unit is simplified as a cube with dimensions of $0.70 \times 0.25 \times 0.30 \mathrm{~m}$ (length $\times$ width $\times$ height). The inlet is at the bottom of the indoor unit, while the outlet is on the top of the indoor unit.

The closed-loop performance of the air-conditioner is compared with three different vane angles and two airflow modes. The vane angle is defined as the angle between the horizontal plane and the up-down flap (Fig. 4). Three vane angles are small $\left(15^{\circ}\right)$, medium $\left(45^{\circ}\right)$ and high vane angle $\left(75^{\circ}\right)$, respectively. Airflow rate of the indoor unit is regulated by the speed of the fan in the unit. The supply air flow rate is $0.08 \mathrm{~m}^{3} / \mathrm{s}$ and $0.15 \mathrm{~m}^{3} / \mathrm{s}$ for low and high fan speeds, respectively. According to Lee et al. (2017), the air flow rate only changes slightly when the vane angle varies since the flow resistance of the up-down flap is very small. In the presented study, therefore, the same air flow rate is used for the same airflow mode regardless of vane angles.

Modelica air-conditioner models define the inlet boundary conditions for the CFD model, i.e., the inlet air temperature and velocity, while CFD calculates the outlet air temperature for the Modelica models. Meanwhile, Modelica models provide the time-average 
surface temperatures of the side walls and the ceiling, and CFD calculates the surface heat flux for the Modelica models. The synchronization time step is 10 sec. The Modelica models are implemented using the Dymola 2019 simulation environment. The cosimulation runs on a desktop with an Intel i7-2600 processor with 8 cores and $8 \mathrm{~Gb}$ of RAM, and the ratio of the physical time to the CPU time is approximately $1: 3$.

Table 1. Gains of PI controllers.

\begin{tabular}{|l|l|}
\hline Parameter & Value \\
\hline$K_{\mathrm{p}}\left(\mathrm{Hz} /{ }^{\circ} \mathrm{C}\right)$ of compressor rpm controller & 2.0 \\
\hline$T_{\mathrm{i}}(\mathrm{sec})$ of compressor rpm controller & 250 \\
\hline$K_{\mathrm{p}}(\mathrm{Count} / \mathrm{K})$ of suction SH controller & 1.25 \\
\hline$T_{\mathrm{i}}(\mathrm{sec})$ of suction SH controller & 66 \\
\hline
\end{tabular}

Table 2. Solver settings and boundary conditions of CFD room model.

\begin{tabular}{|c|c|}
\hline Item & Content \\
\hline $\begin{array}{c}\text { Computational } \\
\text { domain }\end{array}$ & $\begin{array}{c}5 \times 5 \times 2.6 \mathrm{~m} \text { (length } \times \\
\text { width } \times \text { height })\end{array}$ \\
\hline Turbulence model & k-epsilon turbulence model \\
\hline Time dependent & $\begin{array}{c}\text { Transient simulation } \\
\text { (Courant number }<1)\end{array}$ \\
\hline $\begin{array}{c}\text { Inlet boundary } \\
\text { condition }\end{array}$ & $\begin{array}{c}\text { externalCoupledTemperature } \\
\text { \& externalCoupledVelocity }\end{array}$ \\
\hline $\begin{array}{c}\text { Outlet boundary } \\
\text { condition }\end{array}$ & $\begin{array}{c}\text { zeroGradient for temperature } \\
\text { and velocity }\end{array}$ \\
\hline $\begin{array}{c}\text { Side walls \& } \\
\text { ceiling }\end{array}$ & $\begin{array}{c}\text { Isothermal condition } \\
\left(26.85^{\circ} \mathrm{C}\right)\end{array}$ \\
\hline Floor & $\begin{array}{c}\text { fixed temperature gradient } \\
\text { (fixed at } 356 \mathrm{~K} / \mathrm{m})\end{array}$ \\
\hline Solver & buoyantPimpleFoam \\
\hline Scheme & MUSCL \\
\hline
\end{tabular}

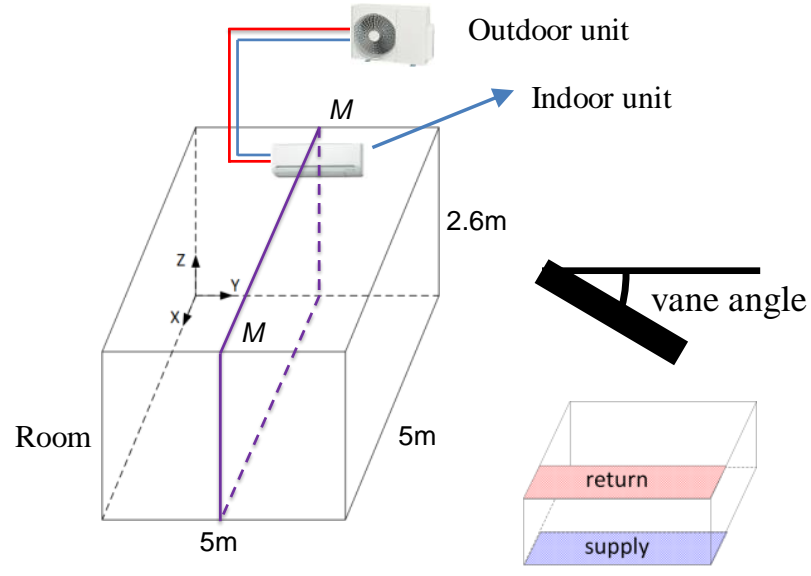

Figure 4. Wall-mounted air-conditioner in a room.

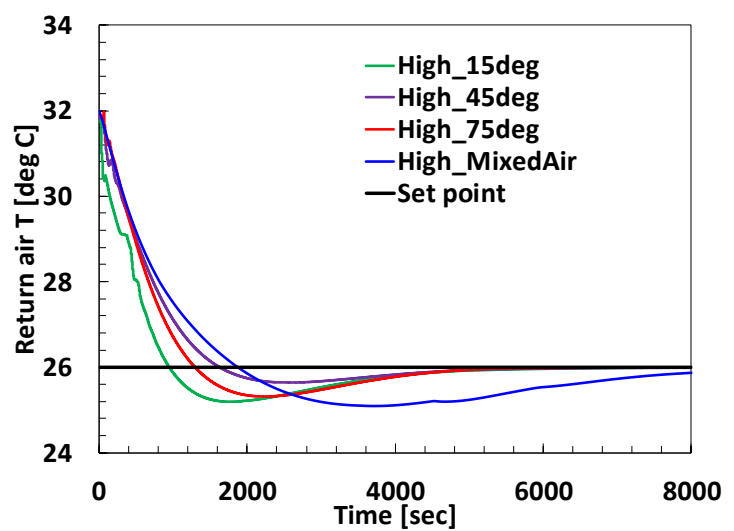

(a)

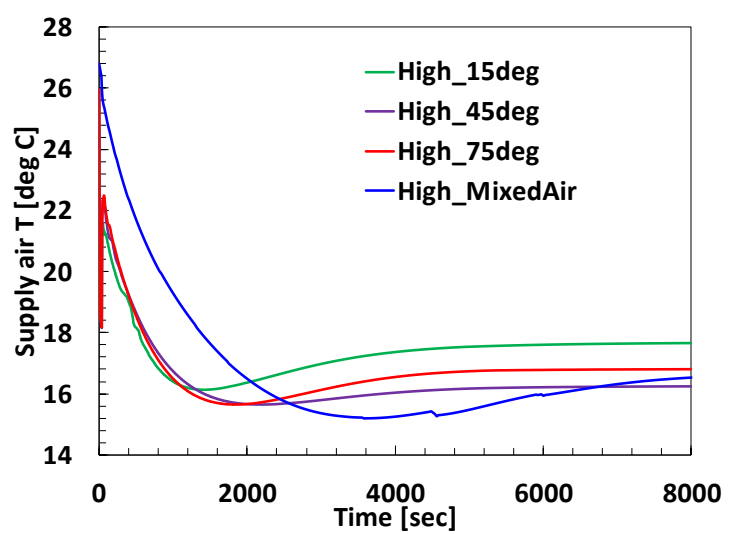

(b)

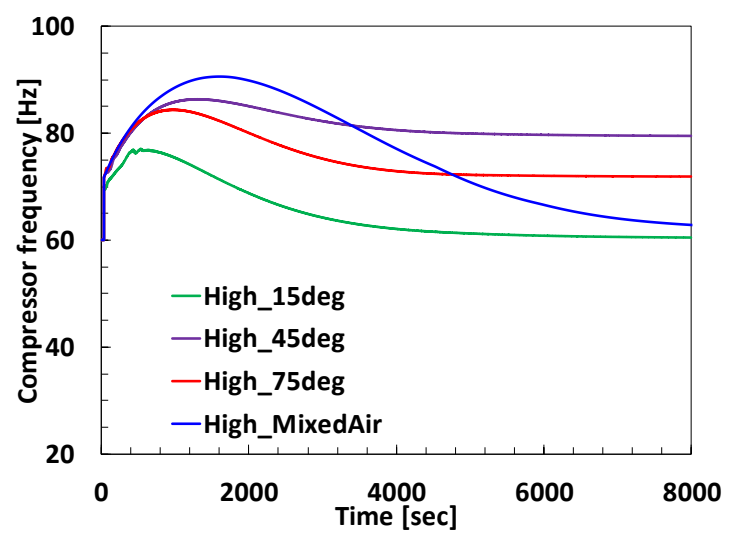

(c)

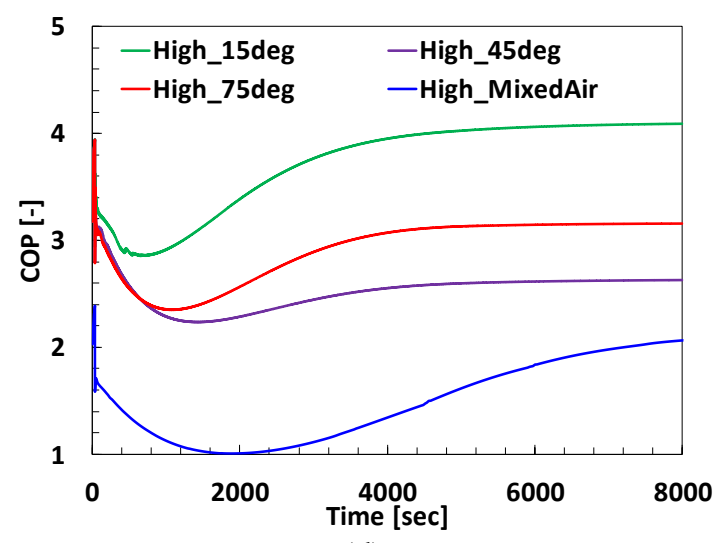

(d)

Figure 5. Dynamics of air-conditioning system in high airflow mode. 


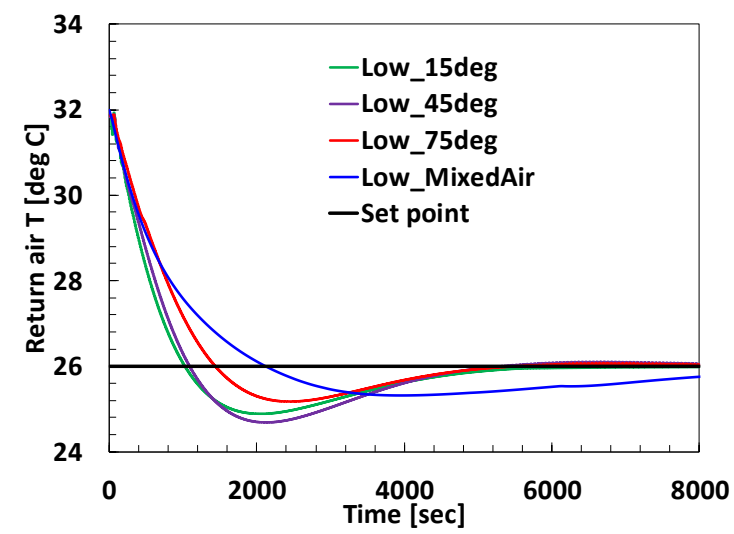

(a)

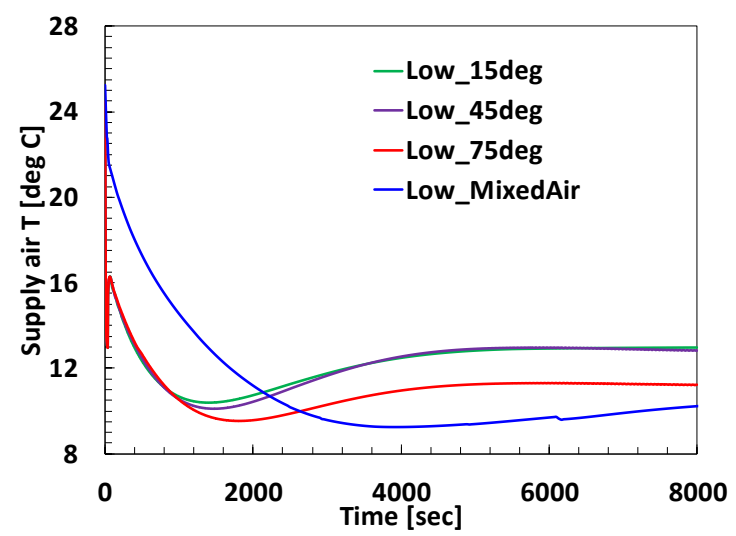

(b)

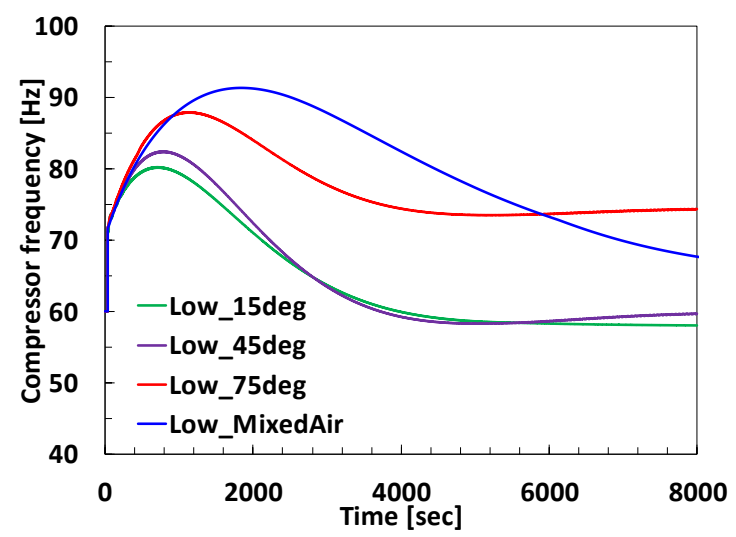

(c)

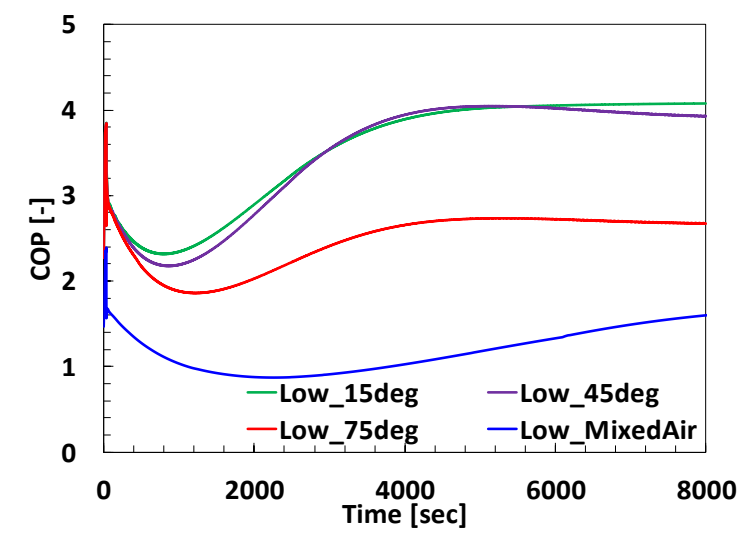

(d)

Figure 6. Dynamics of air-conditioning system in low airflow mode.
Fig. 5 illustrates the dynamic characteristics of return air temperature (a), supply air temperature (b), compressor frequency (c) and coefficient of performance (COP) (d) at different vane angles in the high airflow mode, respectively. It is evident from Fig. 5 a that the vane angle affects the pull-down time, which is defined as the time required to bring down the temperature of room air from the initial temperature to the final desired temperature. The pull-down time for vane angles of $15^{\circ}, 45^{\circ}$ and $75^{\circ}$ is $950 \mathrm{sec}, 1630 \mathrm{sec}$ and $1300 \mathrm{sec}$, respectively. When the vane angle is $15^{\circ}$, the jet flow takes the shortest distance to reach the outlet and entrains the least hot air from the heated floor during the period, resulting in the fastest pull-down. When the vane angle is $75^{\circ}$, the jet flow takes much longer distance to reach the outlet and exhibits a prolonged pull-down time. When the vane angle is $45^{\circ}$, the jet flow reaches the vicinity of the floor center, showing the strongest mixing effect with the hot air on top of the heated floor, resulting in the slowest pull-down. Fig. 5b reveals the transients of supply air temperature and it can be observed that the supply air temperatures vary quite differently for different vane angles under the closed-loop control. Fig. 5c shows the variations of compressor speed during pull-down operation. Given that $15^{\circ}$ vane angle has the best pull-down performance, it is straightforward to expect that this vane angle results in the lowest compressor speed and the highest COP, as shown in Fig. 5 d.

The pull-down transients in the low airflow mode are given in Fig. 6. The pull-down time of the low airflow mode is $1030 \mathrm{sec}, 1090 \mathrm{sec}$ and $1440 \mathrm{sec}$ for vane angles of $15^{\circ}, 45^{\circ}$ and $75^{\circ}$, respectively. In the low airflow mode, the jet flow from the indoor unit has a low momentum and is not affected by the buoyancy easily. Therefore, mixing effect is not as strong as in the high airflow case. Low and medium angles yield shorter pulldown time and higher COP than the high vane angle case because the travelling distance of the jet flow is shorter.

The pull-down transients using the mixed air room model (Wetter et al., 2014) are also given in Figs. 5 and 6. Apparently, the mixed air room model leads to much longer pull-down time and exhibits more damping than the CFD room model. This is because the mixed air model ignores the non-uniformity in the temperature field and assumes the all the heat is picked up by the jet flow, resulting in the larger load on the compressor and higher return air temperature as well as longer pulldown time. This suggests that the well-mixed air model might not be suited for control design and therefore a detailed CFD model is more favorable.

Figs. 7 and 8 show the temperature and airflow distributions of the middle plane M-M of the room when the return air temperature reaches the set point in the high and low airflow modes, respectively. The figures illustrate that air near the inlet has the lowest 
temperatures, whereas air near the walls and floor and has the highest temperatures. The air temperatures clearly are not uniformly distributed as assumed by the well-mixed air model.

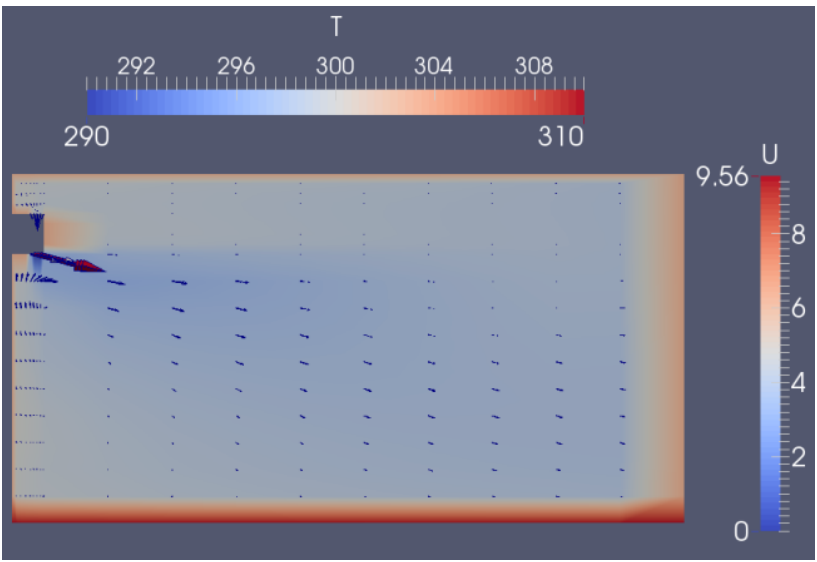

(a)

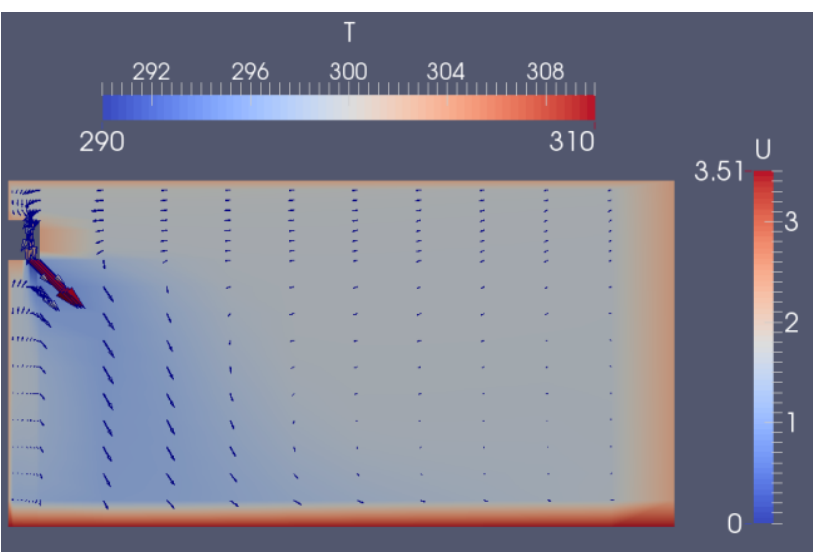

(b)

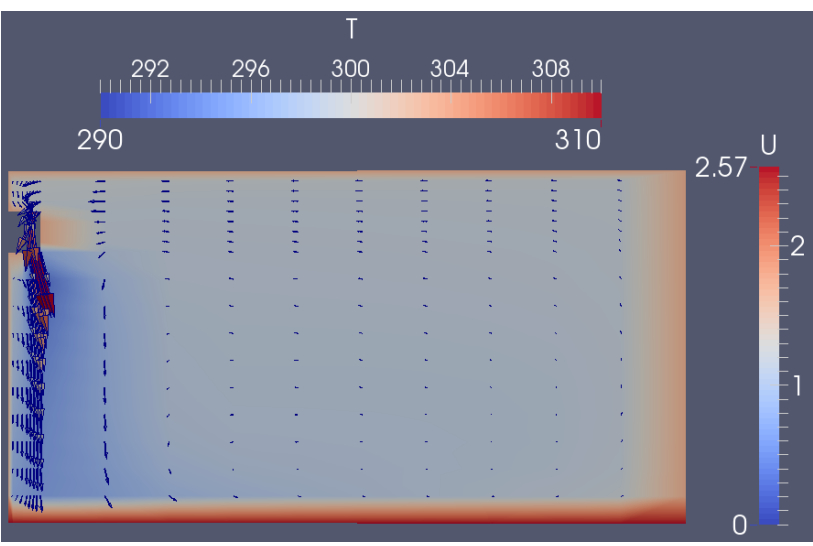

(c)

Figure 7. Temperature and airflow distributions in high airflow mode: (a) $-15^{\circ}$ vane angle; (b) $-45^{\circ}$ vane angle; (c) $-75^{\circ}$ vane angle.

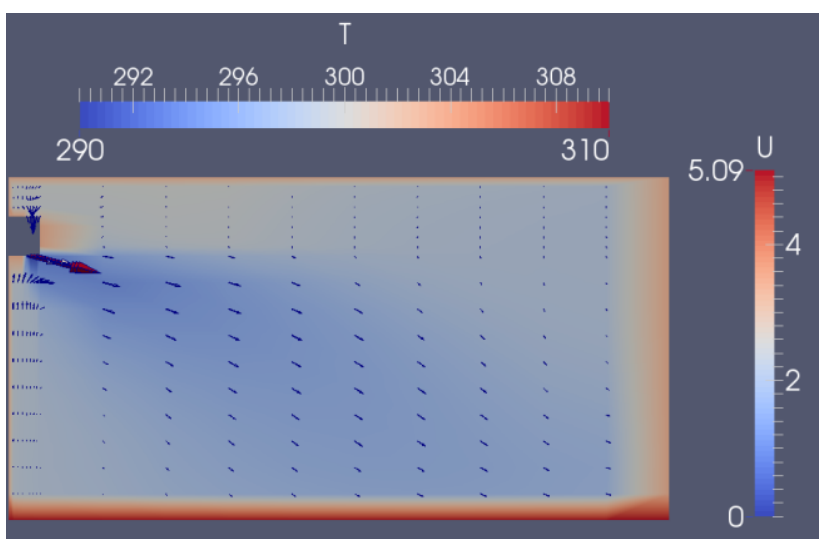

(a)

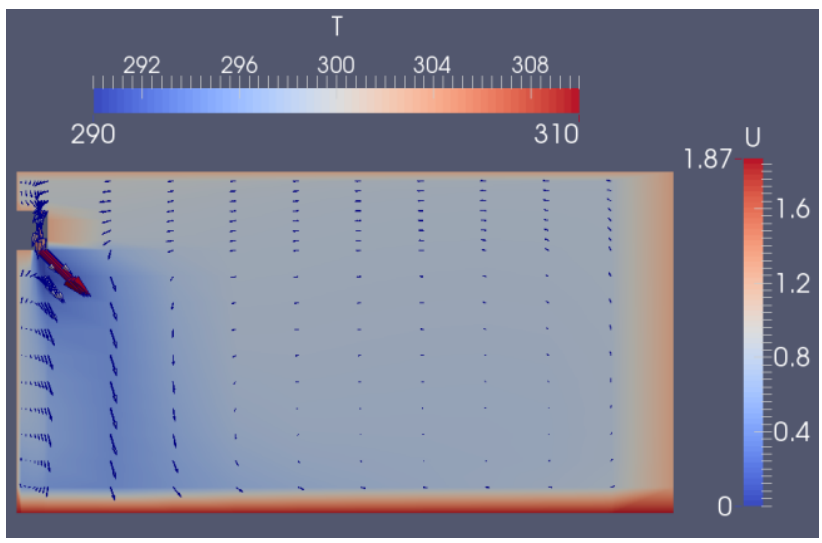

(b)

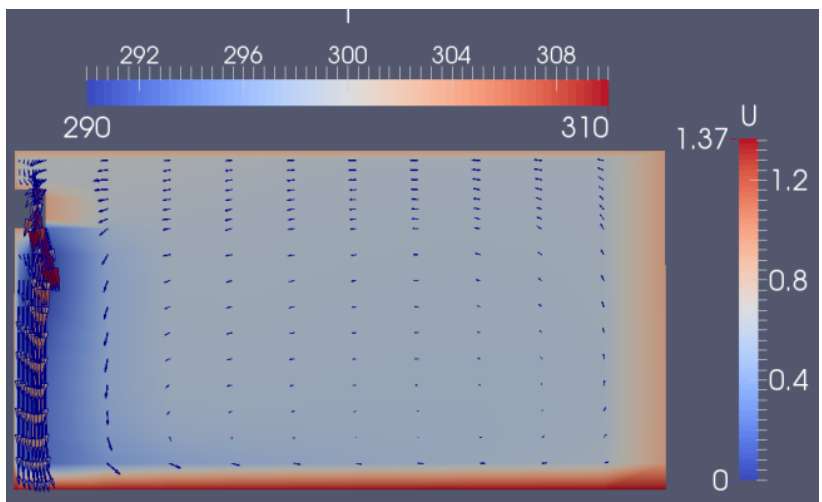

(c)

Figure 8. Temperature and airflow distributions in low airflow mode: (a) - $15^{\circ}$ vane angle; (b) $-45^{\circ}$ vane angle; (c) $-75^{\circ}$ vane angle.

\section{Conclusions}

This paper demonstrated a coupled simulation of Modelica and OpenFOAM for the transient modeling of a wall-mounted split-type room air conditioner during pull-down operation. The use of coupled simulation with detailed CFD model for indoor environment facilitates more accurate exploration of system dynamics than using the well-mixed air model due to the inherent non-uniform air flow and temperature distributions in buildings. Numerical simulations indicated that the vane angle and airflow mode showed 
pronounced impact on the pull-down performance of air-conditioning system. Future work will include explore the effect of location of sensor and heat source as well as experimental validation.

\section{References}

Zhiqiang Zhai, Qingyan Chen, Philip Havesb and Joseph H. Klems. On Approaches to Couple Energy Simulation and Computational Fluid Dynamics Programs. Buildings and Environment, No 37, pp. 857-864, 2002. doi: /10.1016/S0360-1323(02)00054-9.

Wei Tian, Xu Han, Wangda Zuo and Michael D. Sohn. Building Energy Simulation Coupled with CFD for Indoor Environment: A Critical Review and Recent Applications. Energy and Buildings, No 165, pp. 184-199, 2018. doi: 10.1016/j.enbuild.2018.01.046.

Wangda Zuo, Michael Wetter, Wei Tian, Dan Li, Mingang Jin and Qingyan Chen. Coupling Indoor Airflow, HVAC, Control and Building Envelope Heat Transfer in the Modelica Buildings Library. Building Performance Simulation, No 9, pp. 366-381. 2016. doi: 10.1080/19401493.2015.1062557.

OpenFOAM Foundation. https://github.com/OpenFOAM/ /OpenFOAM-dev.

Joost J. Brasz and Kenneth Koenig. Numerical methods for the transient behavior of two-phase flow heat transfer in evaporators and condensers. Numerical Properties and Methodologies in Heat Transfer, pp: 461-476, 1983.

Hongtao Qiao, Vikrant Aute and Reinhard Radermacher. Transient Modeling of a Flash Tank Vapor Injection Heat Pump System - Part I: Model Development. International Journal of Refrigeration, No 49, pp.169-182, 2015. doi: 10.1016/j.ijrefrig.2014.06.019.

Salomon Levy. Forced Convection Subcooled Boiling Prediction of Vapor Volumetric Fraction. International Journal of Heat and Mass Transfer, No 10, pp: 951-965, 1967. doi: 10.1016/0017-9310(67)90071-3.

Sihwan Lee, Juyoun Lee and Shinsuke Kato. Influence of Vane Angle on the Effectiveness of Air Conditioning of Wall-mounted Split-type Air Conditioners in Residential Buildings. Science and Technology in the Built Environment, No 23, pp. 761-775. 2017. doi: 10.1080/23744731.2016.1260410.

Michael Wetter, Wangda Zuo, Thierry S. Nouidui and Xiufeng Pang. Modelica Buildings Library. Building Performance Simulation, No. 7, pp: 253-270, 2014. doi: 10.1080/19401493.2013.765506. 
